\section{Valores de referencia para la batería de pruebas Senior Fitness Test en mujeres mayores chilenas físicamente activas}

\author{
PABLO VALDÉS-BADILLA ${ }^{1,2, a, c}$, YENY CONCHA-CISTERNAS ${ }^{3,4,5, b}$, \\ EDUARDO GUZMÁN-MUÑOZ ${ }^{5, \mathrm{~b}, \mathrm{c}}$, JENNY ORTEGA-SPULER ${ }^{6, \mathrm{a}, \mathrm{c}}$, \\ RODRIGO VARGAS-VITORIA ${ }^{1, \mathrm{a}, \mathrm{c}, \mathrm{d}}$
}

\section{Reference values for the senior fitness test in Chilean older women}

Background: The Senior Fitness Test (SFT) is frequently used to assess physical fitness and functional independence in older people. Aim: To establish reference values for the SFT in Chilean physically active older women according to age ranges. Material and Methods: Cross-sectional study that included 1048 Chilean women aged between 60 and 85 years. Chair stand (CS), arm curl (AC), two-min walk (2 min), chair sit-and-reach (CSr), back scratch (BS), and timed up-and-go test (TUG) were evaluated. The reference values are presented in percentiles ( $p 5, p 10, p 25, p 50, p 75, p 90$ and $p 95)$ and are distributed age intervals (60-64, 65-69, 70-74, 75-79 and $\geq 80$ years). Results: There was a decrease in strength (CS and $A C$ ), aerobic resistance (2 min) and flexibility (CSr and BS) along with age, whereas the time required to perform the timed up and go increased along with age. Conclusions: Physically active older women show a deterioration in physical fitness along with age. These women have higher reference values in CS, AC, 2 min and CSr, and lower in BS and TUG, than those reported abroad for the SFT.

(Rev Med Chile 2018; 146: 1143-1150)

Key words: Aged; Aging; Exercise; Reference Values.
${ }^{1}$ Instituto de Actividad Física y

Salud, Universidad Autónoma de Chile, Chile.

2Programa de Doctorado en Ciencias de la Actividad Física, Facultad de Ciencias de la Educación, Universidad Católica del Maule, Chile.

${ }^{3}$ Universidad Tecnológica de Chile INACAP, Chile.

${ }^{4}$ Programa de Magíster en

Ciencias de la Actividad Física,

Universidad Católica del Maule,

Talca, Chile.

${ }^{5}$ Escuela de Kinesiología, Facultad de Salud, Universidad Santo Tomás, Chile.

${ }^{6}$ Instituto Nacional de Deportes, Región de La Araucanía, Chile. aProfesor de Educación Física.

${ }^{\mathrm{b}}$ Kinesiólogo.

'MsC.

dPhD en Motricidad Humana.

Los autores declaran no tener conflictos de interés. Trabajo no recibió financiamiento.

Recibido el 13 de abril de 2018, aceptado el 27 de septiembre de 2018.

Correspondencia a:

Yeny Concha Cisternas yenyf.concha@gmail.com
E 1 crecimiento acelerado de la población de adultos mayores (AM) proyecta a la región Sudamericana como la de mayor envejecimiento para el año $2050^{1}$. Situación que reviste preocupación en todos los países, especialmente en Chile, debido, entre otras cosas, al aumento del sedentarismo e inactividad física en ese grupo de la población ${ }^{2,3}$. La inactividad física se ha descrito como un factor de riesgo de mortalidad, incluso mayor que el exceso de peso ${ }^{4}$, mientras que mantener una adecuada condición física se transforma en un potente agente protector de la salud ${ }^{5-7}$.

La condición física corresponde a la capacidad fisiológica para realizar actividades de la vida diaria con normalidad, de manera segura, independiente, sin fatiga excesiva y que depende de la indemnidad de aptitudes físicas, tales como; fuerza, resistencia aeróbica, flexibilidad, agilidad y equilibrio dinámico ${ }^{8}$. El déficit en los componentes de la condición física han sido vinculados con discapacidad, mayor riesgo de caídas, fracturas y disminución en la calidad de vida ${ }^{9,10}$.

Habitualmente, los AM son evaluados para determinar su condición física e independencia funcional en centros de salud y organizaciones deportivas a través de pruebas derivadas de la batería Senior Fitness Test (SFT), por permitir una evaluación de gran fiabilidad y fácil aplicación, junto con ofrecer una importante base de datos 
normativos ${ }^{8,11-15}$. Sin embargo, hasta donde llega nuestro conocimiento, la batería SFT cuenta con valores de referencia que no discrimina el nivel de actividad física (AF) de los AM, particularmente, en aquellos que cumplen las recomendaciones internacionales de AF, que señalan $150 \mathrm{~min}$ de AF moderada o 75 min de AF vigorosa a la semana $^{16,17}$.

Por otra parte, $15,8 \%$ de los AM chilenos es físicamente activo, de los cuales, solo $23,6 \%$ corresponde a mujeres ${ }^{3}$. Pese a los bajos niveles de AF manifestados por las mujeres mayores, son ellas las que concentran la mayor participación en iniciativas gubernamentales de AF en programas como: "Adulto Mayor en Movimiento" del Instituto Nacional de Deportes ${ }^{18}$ y el programa "Más Adultos Mayores Autovalentes" del Ministerio de Salud ${ }^{19}$. Además, las mujeres mayores chilenas consideradas físicamente activas manifiestan patrones de rendimiento físico-funcionales superiores a los esperados para su edad y sexo ${ }^{20,21}$.

En este sentido, contar con valores normativos ajustados a los hábitos de AF en mujeres mayores chilenas autónomas, ayudaría a otorgar mayor precisión al diagnóstico, evaluación y seguimiento de su condición física y, con ello, favorecer la prescripción de planes de ejercicio físico individualizados según su capacidad y rango de edad. Hecho reportado en investigaciones previas con AM de Brasil ${ }^{22}$, España ${ }^{23}$ y Portugal $^{24}$, reflejando la necesidad de contextualizar este tipo de datos. De este modo, el presente estudio tiene como objetivo principal establecer valores de referencia de condición física determinados por medio de la batería de pruebas SFT en mujeres mayores chilenas físicamente activas, según rangos de edad. Secundariamente, pretende comparar los valores obtenidos con datos normativos de referencia.

\section{Material y Método}

Estudio transversal que evaluó 1.048 mujeres mayores con edades entre los 60 y 85 años, seleccionadas bajo un criterio no probabilístico intencionado, residentes en dos regiones de Chile: La Araucanía $(\mathrm{n}=518)$ y el Maule $(\mathrm{n}=530)$. Los criterios de inclusión utilizados, fueron: a) practicar regularmente AF moderada al menos $150 \mathrm{~min}$ semanales o vigorosa por 75 min o más por un período mayor a 6 meses $^{16,17,25}$; b) tener entre $60 \mathrm{y}$ 94 años; c) presentar capacidad para comprender y seguir instrucciones; d) ser autónoma. Se consideró autónoma a las mujeres que contaban con un puntaje $\geq 43$ puntos en el examen funcional del adulto mayor (EFAM-Chile) ${ }^{26}$. Fueron excluidas las mujeres mayores que presentaron: a) alguna discapacidad física que limitara su desempeño físico-funcional; b) aquellas que poseían lesiones musculoesqueléticas o en tratamiento de rehabilitación física que impidiera su normal desempeño físico; c) quienes tuviesen contraindicaciones permanentes o temporales para realizar AF. Todas las participantes fueron informadas de los alcances de la investigación y firmaron un consentimiento informado que autoriza el uso de la información con fines científicos. El protocolo de investigación fue revisado y aprobado por el Comité Ético Científico de la Universidad Autónoma de Chile y desarrollado siguiendo lo expuesto en la Declaración de Helsinki.

\section{Mediciones $y$ procedimiento}

Para caracterizar a las mujeres mayores se utilizó: el peso corporal en kilos con balanza mecánica (Scale-tronix, USA; precisión $0,1 \mathrm{~kg}$ ), la estatura bípeda en metros a través de estadiómetro (Seca modelo 220, Alemania; precisión $0,1 \mathrm{~cm}$ ) y el índice de masa corporal (IMC) por medio de la división del peso corporal por la estatura bípeda al cuadrado $\left(\mathrm{kg} / \mathrm{m}^{2}\right)$. Todas las medidas se realizaron de acuerdo a las recomendaciones de la Sociedad Internacional para Avances de la Cineantropometría $(I S A K)^{27}$.

La condición física fue evaluada por medio del protocolo de pruebas SFT, descrito y validado previamente para personas autónomas sin problemas de salud entre 60 y 94 años de edad ${ }^{11}$. El orden de aplicación de las pruebas contempladas en la batería fue: a) prueba de sentarse y levantarse de una silla (SL) para evaluar fuerza de tren inferior, contabilizando el número de repeticiones en $30 \mathrm{~s}$; b) prueba de flexiones del codo (FC) para evaluar fuerza de tren superior sobre la extremidad dominante, utilizando una mancuerna de $3 \mathrm{lbs}$, contabilizando el número de repeticiones en $30 \mathrm{~s}$; c) prueba de dos minutos de marcha (2 min) para evaluar resistencia aeróbica, registrando el número de elevaciones de rodillas que alcanzan, al menos, un ángulo 
de $70^{\circ}$ sobre la articulación coxofemoral de cada participante; d) prueba de flexión del tronco en silla (FTr) para evaluar flexibilidad de tren inferior, medido en cm; e) prueba de juntar las manos tras la espalda (JM) para evaluar flexibilidad de tren superior, medido en $\mathrm{cm}$; f) prueba de levantarse, caminar y volver a sentarse (LCS) para evaluar agilidad y equilibrio dinámico, rodeando un cono a 2,44 $\mathrm{m}$ y registrando el tiempo en segundos.

Las evaluaciones se obtuvieron en tres sesiones: a) en la primera sesión se midieron las pruebas de SL y FC a través de una sola repetición; b) en la segunda sesión fue evaluada la prueba de $2 \mathrm{~min}$ (una repetición); c) en la tercera sesión se midieron la FTr, JM y LCS, cada prueba fue repetida dos veces y se reportó el mejor rendimiento de cada participante. Todas las mediciones se llevaron a cabo en los respectivos centros de salud $\mathrm{u}$ organizaciones deportivas beneficiarias con talleres de AF para mujeres mayores por investigadores entrenados respecto al protocolo de la batería de pruebas SFT.

\section{Análisis estadístico}

Para el análisis estadístico se utilizó el software SPSS 23.0 para Windows (SPSS Inc., IL, USA). La estadística utilizada fue de tipo descriptiva. Se obtuvieron las medias aritméticas y desviaciones estándares de cada una de las variables investigadas. Con el objetivo de utilizar estos resultados como valores de referencia para la condición física de mujeres mayores físicamente activas, los datos obtenidos se presentan en tablas de percentiles (p5, p10, p25, p50, p75, p90 y p95) distribuidos en intervalos de cinco años de edad (60-64, 65-69, 70-74, 75-79 $\mathrm{y} \geq 80$ años).

\section{Resultados}

En la Tabla 1 se presentan las características basales de la muestra. Los valores para el peso corporal, estatura bípeda e IMC fueron similares al distribuir a las mujeres mayores evaluadas de acuerdo a rangos de edad.

La Tabla 2 expone la propuesta de percentiles para la condición física determinada a través de la batería de pruebas SFT en mujeres mayores físicamente activas según rangos de edad. Se advierte que en las evaluaciones de fuerza (SL y FC), resistencia aeróbica (2 min) y flexibilidad (FTr y $\mathrm{JM}$ ), el rendimiento disminuyó a medida que las mujeres avanzaban en edad. Mientras que, en la prueba de agilidad y equilibrio dinámico (LCS) demoraron más tiempo en realizar el recorrido a mayor edad.

Los resultados de condición física obtenidos en nuestro estudio son comparados con los valores propuestos por Rikli \& Jones en la Figura 1. $\mathrm{Al}$ respecto, se observa que las mujeres mayores chilenas físicamente activas alcanzaron un rendimiento físico-funcional superior en fuerza (SL y FC), resistencia aeróbica (2 min) y flexibilidad del tren inferior (FTr) respecto a los datos normativos de referencia. No obstante, su comportamiento en agilidad y equilibrio dinámico (LCS) y en flexibilidad del tren superior (JM) se encontró por debajo de lo establecido.

Tabla 1. Características de la muestra según rangos de edad

\begin{tabular}{|cccccc|}
\hline $\begin{array}{c}\text { Rangos de edad } \\
\text { (años) }\end{array}$ & $\mathbf{n}$ & $\begin{array}{c}\text { Edad } \\
\text { (años) }\end{array}$ & $\begin{array}{c}\text { Peso corporal } \\
\mathbf{( k g )}\end{array}$ & $\begin{array}{c}\text { Estatura bípeda } \\
\mathbf{( m )}\end{array}$ & $\begin{array}{c}\text { IMC } \\
\left(\mathbf{k g} / \mathbf{m}^{\mathbf{2}}\right)\end{array}$ \\
\hline $60-64$ & 211 & $62,1(1,6)$ & $71,8(12,4)$ & $1,53(0,1)$ & $30,6(5,4)$ \\
\hline $65-69$ & 288 & $67,0(1,4)$ & $69,8(12,8)$ & $1,51(0,1)$ & $30,5(5,1)$ \\
\hline $70-74$ & 239 & $72,0(1,4)$ & $68,3(10,3)$ & $1,51(0,1)$ & $30,0(4,1)$ \\
\hline $75-79$ & 213 & $76,8(1,5)$ & $68,9(13,6)$ & $1,51(0,1)$ & $30,2(5,3)$ \\
\hline 80 y más & 97 & $82,3(5,7)$ & $65,5(13,7)$ & $1,49(0,1)$ & $29,3(5,4)$ \\
\hline Total & 1.048 & $70,6(6,2)$ & $69,2(12,4)$ & $1,51(0,1)$ & $30,2(5,0)$ \\
\hline
\end{tabular}

IMC: índice de masa corporal; n: número de casos. Valores expresados en media y desviación estándar. 
Tabla 2. Percentiles para la batería de pruebas Senior Fitness Test en mujeres mayores chilenas físicamente activas según rangos de edad

\begin{tabular}{|c|c|c|c|c|c|c|c|}
\hline $\begin{array}{l}\text { Rangos de edad } \\
\text { (años) }\end{array}$ & P5 & P10 & P25 & P50 & P75 & P90 & P95 \\
\hline & \multicolumn{7}{|c|}{ Sentarse y levantarse de una silla (repeticiones) } \\
\hline $60-64$ & 12,0 & 13,0 & 15,0 & 17,0 & 19,0 & 23,0 & 25,0 \\
\hline $65-69$ & 11,0 & 12,0 & 15,0 & 17,0 & 20,0 & 23,0 & 25,0 \\
\hline $70-74$ & 12,0 & 12,0 & 14,0 & 16,0 & 19,8 & 22,7 & 26,0 \\
\hline $75-79$ & 10,0 & 10,9 & 13,0 & 16,0 & 19,0 & 22,0 & 24,1 \\
\hline \multirow[t]{2}{*}{80 y más } & 9,0 & 10,0 & 13,0 & 16,0 & 18,0 & 21,7 & 23,4 \\
\hline & \multicolumn{7}{|c|}{ Flexiones del codo (repeticiones) } \\
\hline $60-64$ & 15,1 & 19,0 & 21,0 & 24,0 & 28,0 & 32,0 & 37,8 \\
\hline $65-69$ & 15,0 & 17,0 & 20,0 & 24,0 & 28,0 & 31,0 & 35,0 \\
\hline $70-74$ & 15,0 & 17,0 & 19,0 & 23,0 & 26,3 & 31,0 & 34,4 \\
\hline $75-79$ & 15,0 & 16,0 & 19,0 & 22,0 & 26,0 & 31,0 & 35,0 \\
\hline \multirow[t]{2}{*}{80 y más } & 9,7 & 13,3 & 18,3 & 21,0 & 25,0 & 31,1 & 32,1 \\
\hline & \multicolumn{7}{|c|}{ Dos minutos de marcha (número de elevaciones de rodillas) } \\
\hline $60-64$ & 69,4 & 84,1 & 100,0 & 114,0 & 129,8 & 142,9 & 150,0 \\
\hline $65-69$ & 67,5 & 80,7 & 94,0 & 108,0 & 121,8 & 140,0 & 148,0 \\
\hline $70-74$ & 53,7 & 70,3 & 88,0 & 100,5 & 114,8 & 139,1 & 146,0 \\
\hline $75-79$ & 48,9 & 63,5 & 83,5 & 101,5 & 115,3 & 130,4 & 146,0 \\
\hline \multirow[t]{2}{*}{80 y más } & 42,7 & 50,0 & 71,0 & 96,5 & 114,0 & 125,7 & 140,7 \\
\hline & \multicolumn{7}{|c|}{ Flexión del tronco en silla $(\mathrm{cm})$} \\
\hline $60-64$ & $-8,8$ & $-4,0$ & 0,6 & 5,7 & 12,4 & 17,3 & 21,5 \\
\hline $65-69$ & $-8,9$ & $-4,1$ & 0,0 & 3,4 & 10,0 & 15,3 & 19,0 \\
\hline $70-74$ & $-9,4$ & $-5,2$ & 0,0 & 3,5 & 8,5 & 13,3 & 16,0 \\
\hline $75-79$ & $-9,5$ & $-5,2$ & $-0,4$ & 2,5 & 6,0 & 11,0 & 15,5 \\
\hline \multirow[t]{2}{*}{80 y más } & $-9,4$ & $-6,0$ & $-1,1$ & 1,2 & 6,0 & 8,6 & 10,4 \\
\hline & \multicolumn{7}{|c|}{ Juntar las manos tras la espalda $(\mathrm{cm})$} \\
\hline $60-64$ & $-23,7$ & $-18,0$ & $-11,1$ & $-5,0$ & 1,1 & 2,6 & 4,8 \\
\hline $65-69$ & $-24,2$ & $-17,5$ & $-11,1$ & $-5,3$ & 0,0 & 3,0 & 5,5 \\
\hline $70-74$ & $-25,5$ & $-20,3$ & $-14,0$ & $-8,0$ & $-3,5$ & 3,2 & 6,0 \\
\hline $75-79$ & $-28,1$ & $-21,5$ & $-16,6$ & $-9,9$ & $-4,3$ & 3,9 & 6,3 \\
\hline \multirow[t]{2}{*}{ Más de 80} & $-33,0$ & $-25,8$ & $-17,8$ & $-10,1$ & $-6,3$ & 4,5 & 6,4 \\
\hline & \multicolumn{7}{|c|}{ Levantarse, caminar y volver a sentarse (s) } \\
\hline $60-64$ & 3,9 & 4,1 & 4,4 & 5,0 & 5,5 & 5,9 & 6,8 \\
\hline $65-69$ & 4,0 & 4,2 & 4,7 & 5,2 & 5,9 & 6,4 & 7,5 \\
\hline $70-74$ & 4,3 & 4,6 & 4,9 & 5,4 & 6,0 & 6,8 & 7,3 \\
\hline $75-79$ & 4,3 & 4,6 & 5,0 & 5,8 & 6,7 & 7,5 & 8,2 \\
\hline 80 y más & 4,4 & 4,8 & 5,6 & 6,2 & 7,4 & 8,3 & 9,0 \\
\hline
\end{tabular}

P: percentil. Valores expresados en media. 


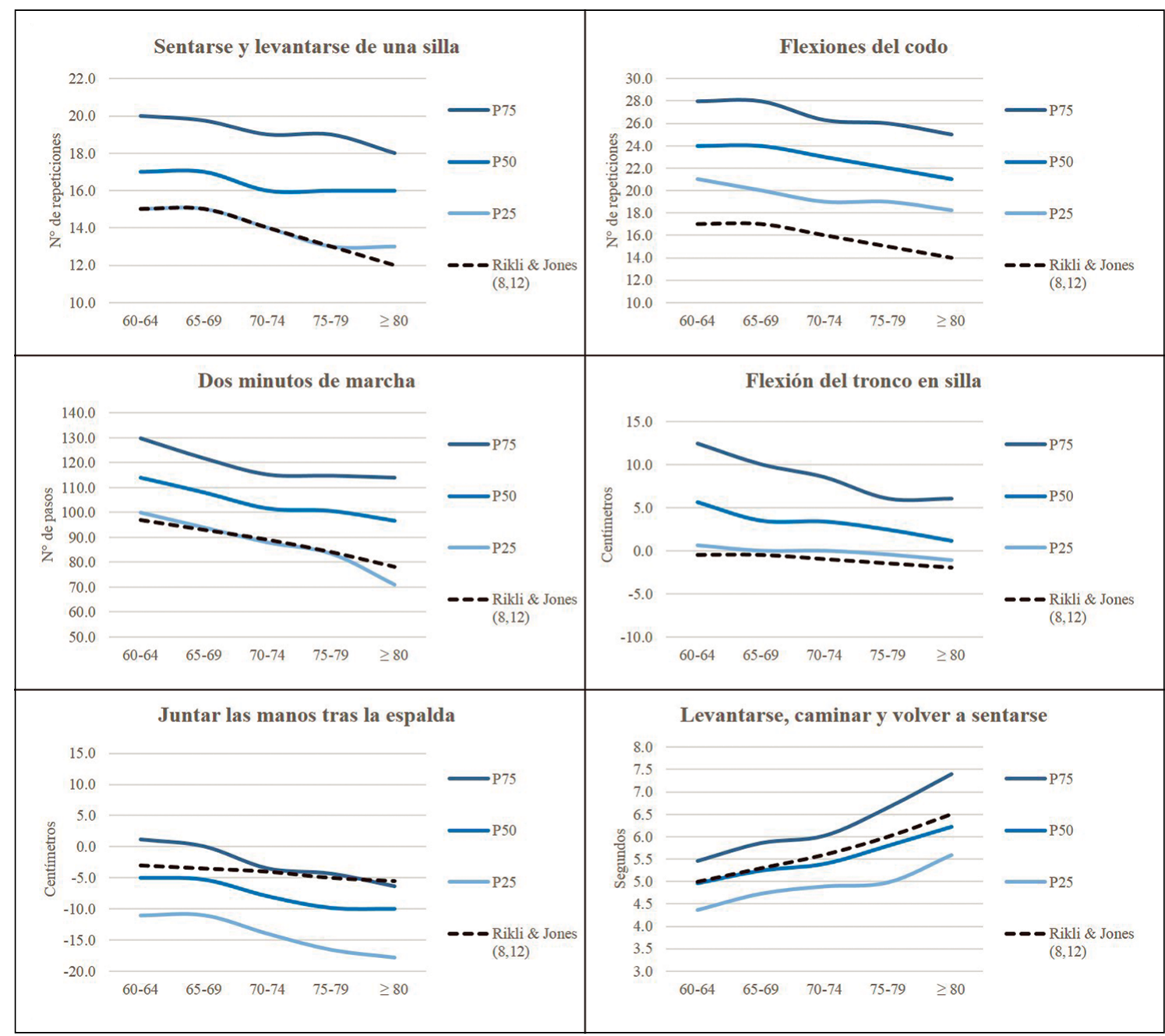

Figura 1. Comparación entre los valores referenciales de condición física con los obtenidos en mujeres mayores chilenas físicamente activas según rangos de edad. P: percentil. Valores de referencia obtenidos de Rikli \& Jones ${ }^{12,28}$.

\section{Discusión}

El principal resultado de nuestro estudio señala que, en general, las mujeres mayores chilenas físicamente activas manifiestan un deterioro de su condición física a medida que avanza la edad. Además, presentan valores de referencia superiores en SL, FC, 2 min y FTr e inferiores, en JM y LCS, respecto a los establecidos para mujeres mayores autónomas, sanas y del mismo rango de edad ${ }^{8,12,28}$. Otras investigaciones también han reportado diferencias en AM de Brasil ${ }^{22}$, Portugal ${ }^{24}$ y España ${ }^{23}$ con los datos establecidos por Rikli \& Jones ${ }^{8,12}$. Hasta donde hemos indagado, este es el primer estudio que presenta tablas normativas para AM que practican AF de forma regular.

Las diferencias en el rendimiento físico-funcional para las pruebas del SFT reportadas por distintas poblaciones, podrían estar relacionadas con los contextos económicos, culturales y sociales propios de cada nación y que, probablemente, influyan sobre la condición física de sus habitantes ${ }^{29}$. Además, los datos de referencia existentes del SFT, en su mayoría, no consideran AM clasificados como físicamente activos ${ }^{8,12-14,22,24,30}$. Estos antecedentes confirman la importancia y necesidad de generar valores de referencia representativos para cada país, y que, además, distingan por nivel de AF. 
Por otro lado, el presente estudio obtuvo el p5, p10, p25, p50, p75, p90 y p95 para cada prueba de condición física del SFT similar a lo realizado en investigaciones con AM de Estados Unidos de Norteamérica ${ }^{12}$, Hong Kong ${ }^{13}$, Noruega ${ }^{14} y$ Portugal $^{24}$. En general, los datos de referencia presentados mediante percentiles permite comparar la condición física de los AM con personas de la misma edad y sexo, lo que ayuda a predecir el riesgo asociado con la pérdida potencial de funcionalidad motriz ${ }^{30}$. Para la fuerza del tren inferior (SL) y superior (FC), resistencia aeróbica (2 min) y flexibilidad del tren inferior (FTr), el rendimiento de las mujeres chilenas fue superior a las recomendaciones entregadas por Rikli \& Jones $^{8,12}$ para mantener un estado de independencia funcional. Esto se atribuye al nivel de AF de las participantes evaluadas. Sin embargo, para la flexibilidad del tren superior (JM), agilidad y equilibrio dinámico (LCS) más de $50 \%$ de la muestra chilena se encuentra por debajo de lo establecido por Rikli \& Jones ${ }^{8,12}$. Estos últimos antecedentes llaman la atención, debido a que los datos internacionales incluyen mujeres con distintos niveles de $\mathrm{AF}^{8,12-14,24}$, mientras que nuestros resultados presentan valores solo de mujeres físicamente activas, por lo cual, se esperaría un rendimiento mayor en nuestras evaluadas. Es probable que el exceso de peso (i.e. IMC 30,2 $\mathrm{kg} / \mathrm{m}^{2}$ ) provoque limitaciones en la agilidad y equilibrio dinámico, mientras que la flexibilidad del tren superior podría estar disminuida por la rigidez de los cartílagos y tejidos que facilitarían la disminución del rango de movimiento articular y redundaría en la disminución de la flexibilidad, hecho reportado en mujeres mayores físicamente activas $^{21}$. Mediciones antropométricas más precisas en mujeres mayores chilenas sería conveniente realizar para poder afirmarlo.

Las mujeres que participaron en el estudio mostraron pérdidas de su capacidad física a medida que avanzaban en edad. Sin embargo, este descenso de la condición físico-funcional fue menor que el informado para mujeres mayores de otros países ${ }^{12,13,22,24}$. Esto es atribuido, principalmente, a la práctica regular de $\mathrm{AF}$, situación coincidente con lo informado en AM físicamente activos de Portugal $^{31}$, quienes conseguían mayor rendimiento en las pruebas del SFT respecto a los AM sedentarios. Además, se ha señalado que los AM que participan de un programa de AF alcanzan resultados significativamente mejores en todas las pruebas relacionadas con funcionalidad motriz ${ }^{20}$.

Otro elemento interesante de nuestro estudio consistió en la tasa de declinación funcional en mujeres entre los 65-69 y 80-84 años. Para la prueba SL, se reporta en Estados Unidos de Norteamérica $16,3 \%^{12}$, en Brasil $17,1 \%^{22}$ y en Portugal $35,5 \%{ }^{24}$, mientras que en nuestros resultados, alcanzaron $7,9 \%$. Comportamiento similar se manifiesta en la FC con una declinación de 14,5\%, $17,8 \%$ y $29,9 \%$ en Estados Unidos de Norteamérica, Brasil y Portugal, respectivamente ${ }^{12,22,24}$. En nuestros resultados, el cambio es solo de 9,6\%. La agilidad y equilibrio dinámico (LCS) presentó, en las mujeres chilenas, una descenso de $17,3 \%$, por su parte Estados Unidos de Norteamérica reporta $22,2 \%^{12}$, Brasil $26,2 \%{ }^{22}$ y Portugal $46,6 \%{ }^{24}$. En cuanto a la prueba de $2 \mathrm{~min}$, la tasa de declinación en nuestras evaluadas fue de 14,7\%, mientras que en Brasil ${ }^{22}$, Portugal ${ }^{24}$ y Estados Unidos de Norteamérica $^{12}$ supera el $19 \%$. Respecto a la flexibilidad, las mujeres chilenas presentaron un descenso de 54,8\% para JM, valores disímiles se exhibieron en Estados Unidos de Norteamérica con $20 \%{ }^{12}$ y España con $83 \%{ }^{23}$. Para la prueba de FTr, las chilenas alcanzaron $73,7 \%$, valor más bajo que el reportado en Brasil y Estados Unidos de Norteamérica $(>150 \%)^{8,22}$. Las diferencias observadas en las tasas de declinación entre los diversos países fortalecen la idea de generar tablas normativas contextualizadas y que consideren las características de sus habitantes, como fue el caso de esta investigación.

Como limitación se podría señalar las características de los AM evaluados, lo que reduce el uso de los valores de referencia con otros grupos de la población. No obstante, nuestros resultados se constituyen en un aporte a los programas de AF enfocados a los AM chilenos.

En conclusión, las mujeres mayores físicamente activas manifiestan un deterioro de la condición física a medida que avanza la edad. Además, presentan valores de referencia superiores en SL, FC, 2 min y FTr e inferiores, en JM y LCS respecto a los reportados internacionalmente para las pruebas del SFT. Por lo tanto, proponemos el uso de los puntos de corte obtenidos en este estudio como valor de referencia para mantener un estado de independencia funcional en mujeres chilenas de acuerdo a su edad y nivel de AF. Asimismo, sería importante considerar la evaluación periódica de 
la condición física en los AM, no solo para otorgar mayor precisión a las intervenciones de AF, sino que también, como una sencilla herramienta de diagnóstico, evaluación y seguimiento de la salud en la vejez.

\section{Referencias}

1. He W, Goodkind D, Kowal P. U.S. Census Bureau, International Population Reports, P95/16-1, An Aging World: 2015 In: U.S. Government publishing office, editor. Washington, DC, 2016. Disponible en: https:// www.census.gov/content/dam/Census/library/publications/2016/demo/p95-16-1.pdf.

2. Ministerio de Salud. Encuesta Nacional de Salud 20162017: Primeros resultados. Santiago, Chile: MINSAL, 2017. Disponible en: http://web.minsal.cl/wp-content/ uploads/2017/11/ENS-2016-17_PRIMEROS-RESULTADOS.pdf.

3. Ministerio del deporte. Encuesta Nacional de Hábitos de Actividad Física y Deportes 2015. Santiago, Chile: MINDEP, 2016. Disponible en: http://www.mindep.cl/ wp-content/uploads/2016/07/PRESENTACION-ENCUESTA-HABITOS-2015.pdf.

4. McAuley PA, Artero EG, Sui X, Lee D-C, Church TS, Lavie CJ, et al, editors. The obesity paradox, cardiorespiratory fitness, and coronary heart disease. Mayo Clin Proc 2012; 87 (5): 443-51.

5. Barry VW, Baruth M, Beets M, Durstine J, Liu J, Blair S. Fitness vs fatness on all-cause mortality: a meta-analysis. Prog Cardiovasc Dis 2014; 56 (4)382-90.

6. Westerterp KR. Daily physical activity and ageing. Curr Opin Clin Nutr Metab Care 2000; 3 (6): 485-8. Disponible en: https://insights.ovid.com/pubmed?pmid=11085835.

7. Goldspink DF. Ageing and activity: their effects on the functional reserve capacities of the heart and vascular smooth and skeletal muscles. Ergonomics 2005; 48 (1114): 1334-51.

8. Rikli RE, Jones CJ. Development and validation of a functional fitness test for community-residing older adults. J Aging Phys Act 1999; 7 (2): 129-61.

9. Maslow AL, Price AE, Sui X, Lee D-C, Vuori I, Blair SN. Fitness and adiposity as predictors of functional limitation in adults. J Phys Act Health 2011; 8 (1): 18-26.

10. Rosengren BE, Ribom EL, Nilsson J-A, Mallmin H, Ljunggren Ö, Ohlsson C, et al. Inferior physical performance test results of 10,998 men in the MrOS Study is associated with high fracture risk. Age Ageing 2012; 41 (3): 339-44.
11. Rikli RE, Jones CJ. Senior fitness test manual. 2nd ed. Champaign: Human Kinetics, 2013.

12. Rikli RE, Jones CJ. Development and Validation of Criterion-Referenced Clinically Relevant Fitness Standards for Maintaining Physical Independence in Later Years. Gerontologist 2013; 53 (2): 255-67.

13. Chung P-K, Zhao Y, Liu J-D, Quach B. Functional fitness norms for community-dwelling older adults in Hong Kong. Arch Gerontol Geriatr 2016; 65: 54-62.

14. Langhammer B, Stanghelle JK. Functional fitness in elderly Norwegians measured with the Senior Fitness Test. Adv Physiother 2011; 13 (4): 137-44.

15. Langhammer B, Stanghelle JK. The Senior Fitness Test. J Physiother 2015; 61 (3): 163.

16. World Health Organizations. Global Strategy on Diet, Physical Activity and Health. Physical Activity and Older Adults. Geneva: WHO, 2010. Disponible en: http:// www.who.int/dietphysicalactivity/factsheet_olderadults/ en/

17. Garber CE, Blissmer B, Deschenes MR, Franklin BA, Lamonte MJ, Lee I-M, et al. American College of Sports Medicine position stand. Quantity and quality of exercise for developing and maintaining cardiorespiratory, musculoskeletal, and neuromotor fitness in apparently healthy adults: guidance for prescribing exercise. Med Sci Sports Exerc 2011; 43 (7): 1334-59.

18. Instituto Nacional de Deportes. (2016). Adulto Mejores. Santiago: Ministerio del Deporte. Disponible en: http:// www.ind.cl/deporte-para-todos/adultos-mejores/

19. Ministerio de Salud. (2015). Programa Más Adultos Mayores Autovalentes. Santiago, Chile: Ministerio de Salud. Disponible en: http://web.minsal.cl/adultomayor_videoconferencias/

20. Concha-Cisternas YF, Guzmán-Muñoz EE, Marzuca-Nassr GN. Efectos de un programa de ejercicio físico combinado sobre la capacidad funcional de mujeres mayores sanas en Atención Primaria de Salud. Fisioterapia 2017; 39 (5): 195-201.

21. Valdés-Badilla P, Godoy-Cumillaf A, Ortega-Spuler J, Herrera-Valenzuela T, Durán-Agüero S, Zapata-Bastías $\mathrm{J}$, et al. Asociación entre índices antropométricos de salud y condición física en mujeres mayores físicamente activas. Salud Publica Mex 2017; 59 (6, nov-dic): 682-90.

22. Krause MP, Januário RS, Hallage T, Haile L, Miculis CP, Gama MP, et al. A comparison of functional fitness of older Brazilian and American women. J Aging Phys Act 2009; 17 (4): 387-97.

23. Marques EA, Baptista F, Santos R, Vale S, Santos DA, Silva AM, et al. Normative functional fitness standards and trends of Portuguese older adults: cross-cultural comparisons. J Aging Phys Act 2014; 22 (1): 126-37. 
24. Gusi N, Prieto J, Olivares PR, Delgado S, Quesada F, Cebrián C. Normative fitness performance scores of community-dwelling older adults in Spain. J Aging Phys Act 2012; 20 (1): 106-26.

25. Guzmán AM, Ricardo YG, Retamozo LM, Soto JM. Etapas de cambio y actividad física en un grupo de adultos de una localidad de barranquilla. Rev salud mov 2011;3(1):1-7. Disponible en: http://publicaciones.unisimonbolivar.edu.co/rdigital/ojs/index.php/saludmov/ article/view/265/262.

26. Ministerio de Salud. Manual de aplicación del examen de medicina preventiva del adulto mayor. Santiago: MINSAL, 2013. Disponible en: http://web.minsal.cl/ portal/url/item/ab1f81f43ef0c2a6e04001011e011907.pdf

27. Marfell-Jones MJ, Stewart A, de Ridder J. International standards for anthropometric assessment. Wellington: ISAK, 2012. Disponible en: https://repository. openpolytechnic.ac.nz/handle/11072/1510.

28. Rikli RE, Jones CJ. Development and validation of a functional fitness test for community-residing older adults. J Aging Phys Act 1999; 7 (2): 129-61.

29. Theou O, Brothers TD, Rockwood MR, Haardt D, Mitnitski A, Rockwood K. Exploring the relationship between national economic indicators and relative fitness and frailty in middle-aged and older Europeans. Age Ageing 2013; 42 (5): 614-9.

30. Adamo DE, Talley SA, Goldberg A. Age and task differences in functional fitness in older women: comparisons with senior fitness test normative and criterion-referenced data. J Aging Phys Act 2015; 23 (1): 47-54.

31. Santos DA, Silva AM, Baptista F, Santos R, Vale S, Mota $\mathrm{J}$, et al. Sedentary behavior and physical activity are independently related to functional fitness in older adults. Exp Gerontol 2012; 47 (12): 908-12. 